\title{
Eating for Eye Health: Engaging patients with dry age-related macular degeneration in community cookery to support lifestyle change and positive health
}

\author{
Rose Gilbert* - Moorfields Eye Hospital NHS Foundation Trust; NIHR \\ Moorfields Biomedical Research Centre Eating for Eye Health Focus \\ Group; and University College London, UK \\ Ann Rawlings - NIHR Moorfields Biomedical Research Centre Eating for Eye \\ Health Focus Group, UK \\ Michael Dixon - University College London; and NHS England (Culm Valley, \\ Devon), UK \\ Ana Rita Gonçalves de Pinho - NIHR Moorfields Biomedical Research Centre \\ Eating for Eye Health Focus Group; and University College London, UK \\ Tadhg Caffrey - University College London, UK
}

\begin{abstract}
There are limited treatment options available upon diagnosis of dry age-related macular degeneration (AMD), a leading cause of blindness in older people, which progressively threatens central vision and quality of life. Community engagement has the potential to support 'positive health' of individuals with untreatable eye conditions. Eating for Eye Health is an award-winning public-engagement project that aims to raise awareness of research suggesting that nutrition might help protect against progression of AMD and to encourage patients to cook and eat antioxidant-rich food in a community environment. The project engaged patients who had a diagnosis of dry AMD through a focus group and a community cookery day organized in partnership with the healthy food outlet, Pod, and the Manor Gardens Community Kitchen Project, Islington, London. A focus group highlighted participants' potential barriers to engagement with research about lifestyle modification and identified that a co-designed community cookery project could help to address unmet needs for support. Individuals with dry AMD reported increased levels of confidence in cooking skills after participating in the community cookery day. The combination of these methods within the context of AMD highlights how a focus on patient needs and expectations can establish and grow mutually beneficial relationships. There is potential for Eating for Eye Health, or similar community kitchen approaches, to be implemented within the community setting through NHS 'social prescribing' initiatives. In conclusion, Eating for Eye Health is unique in its combination of elements of community consultative and collaborative forms of engagement. These methods could be adopted as part of Sustainability and Transformation Plans (STPs) in local health policy development in the community.
\end{abstract}

Keywords: dry AMD, community kitchens, nutrition, health and well-being, social prescribing, consultation, collaboration 


\section{Key messages}

- Dry age-related macular degeneration (AMD) is a leading cause of untreatable, progressive central vision loss, social isolation and disability in older people.

- Engaging older people with diet through community kitchen initiatives is a promising approach to reducing social isolation and potentially protecting against dry AMD progression in this population.

- Eating for Eye Health is an example of a participant-informed community kitchen intervention, utilizing a combination of elements of community consultative and collaborative forms of engagement, to implement dietary behaviour change and to promote health in the older adult population.

\section{Introduction}

Age-related macular degeneration (AMD) is an eye disease caused in older people by a deterioration of the cells within the central 'macula' area of the retina. It is estimated that one in ten people over the age of 65 have some degree of AMD, and the number of diagnoses is on the rise. The 'dry' form of AMD currently has no treatment and progressively threatens the central field of vision, which has detrimental consequences for everyday activities, such as reading and recognizing the faces of other people. A diagnosis of dry AMD, therefore, has devastating and far-reaching consequences for both individuals ('patients') and their families. However, AMD is still considered a low priority when it comes to publicly funded research and investment. Professor Carrie MacEwan, former President of The Royal College of Ophthalmologists, says:

AMD is the commonest cause of blindness in the developed world and the numbers of patients affected will continue to grow. It must be considered a research priority in order to identify ways to prevent the development of AMD and to treat those affected as early and as effectively as possible. Loss of vision is associated with falls, depression and loss of independence - especially in the older age groups. (VisionUK, 2016)

The symptoms of dry AMD are insidious, and may start with difficulty in reading small print or writing. In the early phase, patients may attribute these difficulties to the wrong glasses prescription, or even brush them off as a normal part of 'getting older'. The diagnosis of an untreatable and progressive sight condition can come as quite a shock and leave people at a loss about how to cope. In the clinical setting of an NHS hospital, doctors are often limited to giving lifestyle advice about eye protection or explaining that dry AMD may further progress to the wet form of AMD. Treatment is available for the wet form of AMD through regular drug injections to the eye (but not the dry form, as previously mentioned), and these often improve vision but are still not curative. For many, this is a frustrating situation. Clinicians are conscious of the adverse impact of AMD, but sometimes fall short of communicating advice that might help support patients through the aftermath of this diagnosis. Patients do not always feel confident in speaking up about their concerns, particularly in a busy clinic. Those with untreatable eye conditions, such as dry AMD, are often discharged from hospital follow-up, with advice to self-monitor their condition and to 'come back if it gets worse'. This approach may leave patients feeling, at best, anxious and, at worst, literally and metaphorically 'left in the dark' by health-care professionals. 
Raising awareness of the research suggesting that lifestyle modifications might benefit eye health is, therefore, important. However, simply passing on an information leaflet to patients is not an adequate solution; it is also crucial to offer support and encouragement to patients in implementing these changes into their lives, particularly in the context of an eye disease that causes progressive deterioration of vision. Modes of community engagement, which have a focus on moving outside of standard modes of dissemination and communication, open up avenues for meaningful discussion about patient needs. These needs relate to the above adverse impacts, but also to the importance of understanding the relationship between patients and their conditions. This complex issue of identity required a novel engagement approach to establish useful conversation and to move forward.

\section{The rationale for Eating for Eye Health}

Evidence from a large clinical trial suggests that antioxidant vitamin supplements may help protect against the progression of AMD (Age-Related Eye Disease Study Research Group, 2001). However, there has been much debate among experts about evidence supporting the recommendation of 'eye vitamin' supplements to prevent or improve eye disease (Evans and Lawrenson, 2017a, 2017b). This has resulted in uncertainty and even scepticism among eye-care professionals about advising patients on nutritional approaches for managing dry AMD. The existing evidence, which provides some support for the inclusion of extra vitamins and minerals in the diet, albeit through supplements, in combination with more recent epidemiological research about the relationship between dietary content and progression of eye disease (Wu et al., 2015; Hogg et al., 2017), certainly raises the strong possibility that a dietary intervention, based on healthy food, may be a feasible strategy for preventing progression of dry AMD. The science behind nutritional approaches to eye health is emerging at a time when nutrition and diet are enjoying much attention in popular media. While information in newspapers, magazines or on the internet might be misleading or inaccurate, it is readily available and communicated in an understandable way to various publics. This motivated the idea of an engagement project to facilitate connection and twoway dialogue about food for eye health, between health professionals with specialist knowledge and those who actually have experience of living with dry AMD.

Following a successful bid for funding and support from UCL Culture, Eating for Eye Health was initiated to engage people with dry AMD, in addition to their friends and relatives, with the current scientific research on nutrition and eye health, to identify specific areas of unmet need and to create a community to address these needs. This was to be achieved through supporting patients in cooking and eating antioxidant-rich food that could support their eye health, in a community setting. We recognized that the benefits of using food as an engagement tool could extend beyond healthy eating and nutrition. Previous social projects have employed food as a tool for community engagement; for example, a 'democratic' communal table was used to facilitate the breaking down of hierarchies and act as a leveller to encourage conversation between community residents (Coulson, 2014; Anchor \& Magnet, 2012). With Eating for Eye Health, we aimed to achieve something similar: a breaking down of hierarchies between patients with AMD and health-care professionals/researchers. 


\section{Project aim}

The aim of the Eating for Eye Health project was to raise awareness of research that suggests nutrition may help protect against the progression of dry AMD, and encourage older people to cook and eat food that could support eye health as part of a local community or social environment.

It is now commonly accepted that health and well-being are closely interlinked, but this concept is not well supported by the traditional medical model. The biomedical perspective on health has typically been disease-focused and based on a scientific process involving observation, description and differentiation, which moves from recognizing and treating symptoms to identifying disease aetiologies and developing specific treatments (Clare, 2001). This viewpoint has been criticized as mechanistic and reductionist, with illness understood in terms of causation and remediation, in contrast to holistic and social models. The World Health Organization (WHO) defines health as 'a state of complete physical, mental and social well-being' and 'not merely the absence of disease' (WHO, 2019: n.p.). Researchers from the Louis Bolk Institute in the Netherlands felt that the WHO definition was inadequate, and developed a new definition of health 'as the ability to adapt and to self manage, in the face of social, physical and emotional challenges' of life (Huber et al., 2016: 1). Health improvement or 'positive health', which takes into account an individual's psychosocial well-being, in addition to their physical health and body functioning, may be targeted through community engagement. The National Institute for Health and Care Excellence (NICE) has noted that, 'Community engagement is a highly complex area with several important purposes. These include empowering people within communities to gain more control over their lives and to play a part in decisions that affect their health and wellbeing' (NICE, 2016: 19).

Further to the direct aims linked to the research and involved groups, we wanted to test how we could bring together different elements of community engagement, concepts of social prescribing, and collaborative engagement in order to bring a group together that would be self-sustaining and enthused to speak about their conditions and identity.

\section{The patient voice in ophthalmology}

There is a tendency for health-care professionals to engage with patients on their terms, rather than those of the patients. It was recognized in the eye-research community some time ago that, although the aim of research into the prevention and treatment of eye disease is to improve the health and quality of life of patients, the people affected by eye disease ('consumers') were seldom involved in the decisions that were made when designing and conducting research (Twamley et al., 2011). Since then, there has been a slowly growing appreciation among eye-care professionals and researchers that the patient is uniquely placed to understand the impact of disease, and can use that position to transform ophthalmic care at the individual and collective level, from research to delivery of care (Dean et al., 2017). Numerous initiatives now exist to support patient and public involvement (PPI) in eye research. For example, the James Lind Alliance (JLA) priority-setting partnership, led by the eye-research charity Fight for Sight (UK), has resulted in an extensive set of unanswered research questions prioritized by patients, as opposed to eye-care professionals (ibid.).

A public-engagement approach, however, has some advantages in that it simultaneously captures the patient and the public voice in its inherent appreciation 
that patients are part of the 'public' community. Public engagement seeks to facilitate public conversations that are not wholly shaped by a 'research agenda', as is often the case in PPI. Furthermore, public engagement is more likely to foster peer-to-peer support within, and communication between, communities due to its preferential focus on the interface between research and wider society. It was thought that opening up an opportunity to engage through cooking and eating would be key to encouraging the creation of a community, and to forging strong relationships between researchers and patients.

In order to gauge interest and inform any plans for a nutritional or cookery intervention for eye health, we hosted a focus group at Pod, a healthy food chain with a branch at Old Street, near Moorfields Eye Hospital, London. Participants were invited to attend via the mailing lists of the Macular Society (a charity with a focus on individuals and their carers affected by macular eye disease and sight loss) and the National Institute for Health Research Biomedical Research Centre at Moorfields Eye Hospital NHS Foundation Trust and UCL Institute of Ophthalmology (NIHR Moorfields BRC). There were 12 attendees, 10 of whom had dry AMD. The discussion revealed that many participants felt 'confused' by what they heard in the media about nutrition, and many were not aware that the food they were eating could influence their eye health. We heard that the eye professionals previously encountered by participants had not explicitly mentioned nutrition being relevant to eye health, and even those focus group participants who had a pre-existing general interest in nutrition did not feel comfortable initiating this discussion in a hospital setting. Some participants did feel that the advice to eat 'colourful, antioxidant-rich' food to benefit their eyes seemed like 'common sense', but still wanted specific advice on which foods to eat. They all expressed a desire for more hospital-led support with their condition, and assistance with achieving the quantity, quality and variety of healthy food to benefit eye health.

Curious about the participants' perceived barriers to healthy eating, we asked them to tell us more about their personal experiences with the practical aspects of nutrition and cooking. The challenges described included: lack of confidence in food preparation, including the use of varied ingredients or unfamiliar foods; perceived high cost associated with good-quality, fresh food and time required for cooking from fresh ingredients; and specific problems with cooking and eating certain foods, such as 'how to cook fish' (concerns about finding recipes and, for some, the 'fishy smell' and 'small bones'), and the struggle to cut up a whole butternut squash ('how should we tackle the hard skin?'). When prompted, some participants specifically spoke about the challenges of cooking with visual impairment, such as their difficulty with reading small print on food packaging, only partially helped by reading or magnifying glasses, and further complicated by a tendency for lenses to 'fog up' with steam from cooking. This opened up a more poignant conversation about guilt with regard to asking for help with 'basic' daily activities such as cooking, the fear of 'becoming a burden' to loved ones, and, significantly, a sense of isolation, hopelessness or even shame, which could be brought up by the experience of living with an untreatable, progressively sightthreatening eye condition. The latter findings are consistent with large research studies demonstrating that irreversible vision loss may prevent individuals from engaging in their daily functional activities and social interactions, and may be predictive of a range of poorer outcomes in later life, including depression (Steinman and Vasunilashorn, 2011; Dagnelie, 2013; Matthews et al., 2017; Tolman et al., 2005). Participant responses also emphasized concerns that there may be a high prevalence of untreated depression in individuals with low vision (Nollett et al., 2016). Depressive symptoms and loneliness could potentially exacerbate a situation in which many individuals with AMD have 
already been shown to be under-consuming nutrients considered to be useful for their condition (Stevens et al., 2015).

Focus group participants were asked whether they would be interested in participating in an educational social event involving cooking for eye health. The idea of a cookery day was perceived by participants to be an original, and a potentially enjoyable, approach to self-care for eyes. A few participants said that they would be happy to attend a community cookery day, but 'would be more interested in eating than cooking'. All participants agreed that they thought that an event with food and/ or cookery as its main focus would be a positive way to engage with eye health. The overall conclusion of the focus group was that initiating a novel engagement approach to nutrition for eye health would be key to encouraging the creation of a community, and to forging a strong relationship between eye professionals, researchers and patients.

\section{Community cookery for improving nutrition and social engagement: Implications for health policy}

It is well recognized that social engagement improves resilience and supports healthy aging (Ozbay et al., 2007; Mendes de Leon, 2005). Research also suggests that it is possible to reduce loneliness by using educational interventions focused on the maintenance and enhancement of social networks (Cohen-Mansfield and Perach, 2015). Community kitchens have the potential to improve social interactions and nutritional intake of participants and their families (Herbert et al., 2014; lacovou et al., 2013), in addition to having sustained impacts on family systems and food purchasing behaviours (Herbert et al., 2014). Exploring the use of community kitchens methodology as part of public engagement has the potential to contribute a unique perspective on the interplay between food, communities and the health of individuals. Researchers, including a group at the UCL Institute of Epidemiology and Health, are currently investigating how best to evaluate the impact of these community kitchen projects on participants.

An innovative way to operationalize community engagement within the NHS framework is through 'social prescribing' initiatives (Kimberlee, 2016, 2013; Brandling and House, 2009). These are sometimes referred to as 'community referral', and are a means by which health professionals in the primary care setting may refer patients to a range of local, non-clinical services. Through recognizing that people's health is determined primarily by a range of social, economic and environmental factors, social prescribing seeks to address people's wider health needs in a more integrated way. It may also support individuals in feeling a greater sense of agency regarding their health conditions. Holistic social prescribing has been shown to be beneficial to supporting patients' health and well-being, as well as to reducing demands on GPs and hospitals (Polley et al., 2017; Kimberlee, 2016).

\section{Co-design of a pilot community cookery intervention}

Feedback from the focus group, expert advice from the Macular Society and the NIHR Moorfields BRC, and a literature review initially informed the development of a pilot intervention: a community cookery day tailored to supporting participants with dry AMD in their dietary behaviours and general well-being. The intention was to combine an approach to consulting with patients (asking them about their condition) with collaborative engagement (working together to cook something) through the creation 
of a community centred around nutritious food, with a shared purpose of improving eye health. The intervention component, focused on encouraging participants to make sustained dietary changes to benefit eye health, was developed according to the COM-B (capability, opportunity, motivation and behaviour) model of behaviour change (Michie et al., 2014; Michie et al., 2011). In contrast to social cognition models, which focus on individual social cognitions, the COM-B model considers individuals within an environmental system, involving the interacting model components of capability, opportunity, motivation and behaviour. The overall approach emphasized the importance of building a community through facilitating a conversation about mutual need and purpose, which would allow co-design of an effective and sustainable intervention for eye health, and subsequent implementation through a social prescribing policy framework (Gilbert et al., 2018).

Qualitative analysis of the focus group discussion transcripts, using the COM-B framework, identified that the group was highly motivated, so it was determined that a co-designed behavioural intervention should target increasing participants' capability and opportunity (that is, the motivation domain was not specifically targeted in this intervention). The COM-B diagnostic tool (Michie et al., 2014) was then used to identify what needed to change in order for focus group participants to make an effective behaviour change towards healthy cooking and eating. Targeting physical and psychological capability suggested that interventions that aimed to increase participants' knowledge, skills and self-efficacy (confidence) regarding eating for eye health, in addition to providing individualized physical assistance, might be effective in implementing dietary behaviour change. Targeting opportunity suggested that interventions that would increase social opportunity, provide the necessary materials at a low cost and support participants in behaviour change would be effective.

At this stage of the project, a potential key challenge was highlighted about the extent to which visual impairment in older people might confer limitations on physical capability for cooking, and the potential health and safety implications of these limitations. It was recognized that, although a hospital or university may have specialized domain expertise in eye disease, there was a paucity of knowledge about the provision of support for day-to-day function and the practical aspects of coping with visual disability. Our perception from the focus group discussion was that the sightimpaired individuals, all of whom had expressed interest in participating in a cookery day, had a good level of self-awareness about their physical capabilities and had already made adaptations to cope with their eye condition. However, we were unsure about whether it would be necessary to make adaptations to the community kitchen space and/or equipment to accommodate sight-impaired individuals, who would be undertaking the cookery activity outside their usual environment. The issue was initially addressed through a consultation with the Macular Society, to find out about their experiences of working with sight-impaired individuals, and how we might determine specific individual requirements for physical assistance with cooking for Eating for Eye Health. We were reassured by the Macular Society that vision impairment need not be a barrier to participation in the cookery activity, and it was further suggested that we could make contact with registered participants individually prior to the day and give them an opportunity to discuss their requirements in confidence. Therefore, registered participants were contacted by email and invited to share any particular expectations or personal needs regarding their attendance at a cookery event by email or telephone. Interestingly, there was no response to this invitation.

In order to further determine the practicalities of designing a cookery day for older people, we researched local initiatives and reached out to Siobhan Mannion, 
who was identified as the manager of an existing community kitchen project at the Manor Gardens Health and Community Centre in our local borough of Islington, London. We found out that Siobhan had been managing an NHS-funded project to support nutritional intake and cooking skills of older people in the local council sheltered housing, but that due to budget constraints, the funding for the project was to be discontinued in order to focus on mental health initiatives in the borough. We recognized that Siobhan would be able to provide valuable expertise and insights into the 'nuts and bolts' of running a community kitchen project for older people, and so we invited her to join the project as an external consultant and operations manager of the pilot cookery day.

We had previously invited focus group participants to send us their healthy recipe ideas for a cookery day (and had received a recipe for preparing dried-fruit 'leather' snacks, which was shared with the group), but we were time-limited in our ability to crowdsource recipes that could be prepared and eaten during a pilot community kitchen session. Therefore, recipes from the 'tried and tested' Manor Gardens Community Kitchen Project portfolio were selected by the project team according to their nutritional content and perceived appearance and taste for our target audience, in combination with specific requests put forward by the focus group, for example, 'ideas for cooking with oily fish'. These recipes were further adapted to enhance their nutritional content and absorption to benefit dry AMD, based on existing clinical evidence and the advice of a clinical research nutrition consultant from the Faculty of Food and Nutrition Sciences, University of Porto, Portugal (who was undertaking concurrent dietetic support work at Barts and the London NHS Trust). A provisional three-course menu was proposed for the cookery day: butternut squash, turmeric and brazil nut soup; salmon fillet with red onion and orange, green and yellow peppers, baked sweet potatoes, and spinach, orange and linseed salad; and apple and blackberry sponge pudding. This was circulated among the focus group participants for their feedback and approval, and we received positive responses ('yum', 'sounds delicious!').

Thus, the focus group participants, eye charity and community kitchen project consultation contributed to the co-design of a community cookery day to which the focus group participants, in addition to others from the original mailing lists of individuals affected by dry AMD, were invited.

The cookery day was held at St Luke's Community Centre, near Moorfields Eye Hospital. There were 12 attendees, including 8 from the original focus group, who participated in a four-hour cookery and food education activity, loosely styled according to the popular, long-running BBC television programme Ready, Steady, Cook. The day was led by Siobhan, an experienced community kitchen manager, and delivery was supported by a team of three postgraduate research students from UCL Institute of Ophthalmology, a research assistant from the NIHR Moorfields BRC and our project nutritionist. The session opened with group 'food bingo' and 'ice-breaker' activities, an explanation of how the day would be organized, and a basic food hygiene and safety 'briefing', all accompanied by copious cups of tea, before participants were divided into smaller groups in order to prepare contributions (for example, fish, soup, salad) to the three-course meal. Prior to the start of the cookery activity, participants were asked to review the three-course menu and self-rate their confidence (on a scale of 1 to 10 , with 1 being least confident and 10 being most confident) in preparing it. They were then assigned to a cookery activity, with attention to their stated needs and preferences. During the activity, there was an opportunity for knowledge exchange between participants and the project team, and the team was also on hand to provide 
assistance to participants where required (although no subjective or objective difficulties were reported). While the food was cooking, we talked through some of the eye-health benefits of common foods, and also invited participants to taste a small selection of popular so-called 'superfoods' that they may not have encountered before, including almond nut butter, sauerkraut and dark chocolate with cocoa nibs.

At the end of the cookery session, we all sat at a communal table to eat the three-course meal together, and talked about the nutritional benefits of each course. Participants were requested to rate each course for taste acceptability, by holding up a 'thumbs up' or 'thumbs down' sign. The feedback was positive, with all participants rating the food as acceptable in taste. All reported increased 10-point-scale confidence scores after completing the cookery activity, indicating that they felt more confident about their cooking skills for recreating these antioxidant-rich recipes at home. 'Eat colourful!' became the project's take-home message. Thematic analysis of qualitative written feedback, both immediately and one week post-intervention, identified positive subjective experiences of 'social participation' and 'peer support'. For example, participants 'enjoyed the sense of community with others who shared their experiences of coping with dry AMD'. Overall, evaluation findings from Eating for Eye Heath were in keeping with published evidence that community kitchen programmes have positive effects on participants' cooking skills confidence, and reduce barriers of cost, waste and knowledge (Garcia et al., 2017). In particular, we received positive feedback, both from participants and academic groups, on our 'practical' and 'engaged' approach to tackling the problem of unmet need within the dry AMD community. This project is further evidence of the importance of flexibly using engagement approaches, and the strong potential to work across a myriad of modes to put the patient voice at the centre of research.

\section{Conclusions}

In summary, successful engagement with the dry AMD community meant Eating for Eye Health was not only a project about engaging participants with research on how to 'eat right for their sight'; it also opened a conversation regarding their unmet needs for psychosocial support. It highlighted the importance of teamwork and communication in creating successful partnerships between health-care professionals, patients and local communities. It was instructive for us to work with and alongside various different agendas, including public engagement, community engagement, PPI and social prescribing in forming this project. We tested the use of mechanisms within these areas with the patient and academic groups, and found them to be responsive when given the tools to articulate themselves in a novel environment. The community kitchen approach was a successful context to bring these elements together, and the result was a richer conversation about identity, lifestyle and disease. Interestingly, ophthalmology patients from a previously published focus group study emphasized their expectations related to communication and interpersonal relationships with their health professionals over and above technical medical interventions, for example prescription medication and diagnostic testing (Dawn et al., 2003). This project certainly highlighted the value of engaging with patients beyond the eye clinic to understand the social impact of chronic eye problems and to better assist them in developing selfcare skills to adapt to their changing landscape.

An important limitation to extrapolating too many conclusions from our engagement approach is that this pilot project involved a small sample of motivated individuals with dry AMD, who had pre-registered their interest in involvement with eye 
research or related projects. Therefore, our findings are not necessarily applicable to a wider or more diverse older population with vision impairment. Community kitchen interventions are potentially expensive and challenging to scale. The success of the project appeared to be related, at least in part, to the small group size, which allowed us to tailor our approach to individual participants. Individuals who participate in clinical and health studies tend to be of higher socio-economic and educational status, and more motivated to change their behaviour and to improve their health, compared with those who do not participate, which potentially perpetuates health inequalities. There is also the challenge of measuring the impact of the lifestyle intervention on altering the course of a disease, which requires precise surrogate endpoints, and are likely to require monitoring over a long period of time in order to see an effect (often much longer than required with a biomedical or pharmaceutical intervention). The next stage of the project could involve a 'needs assessment' of different patient populations with visual impairment; an investigation into how to expand the community kitchens project and/or improve outreach to disadvantaged groups; or an evaluation of social, economic and health impacts of community kitchens and social prescribing on participants.

We feel that findings from this project have the potential to contribute to the growing body of evidence suggesting that community engagement and social prescribing can positively impact health and well-being. Through the creation of a community, it is also hoped that awareness will be raised about AMD, which could attract public funding to this area and facilitate engaged research into improving clinical outcomes, including positive health.

For a summary video, podcast, recipes and further information, please visit the project webpage: https://moorfieldsbrc.nihr.ac.uk/involving-people/involvementexamples/case-study-eating-for-eye-health/eating-for-eye-health-engagement.

\section{Acknowledgments}

Eating for Eye Health was funded and supported by a Beacon Bursary from UCL Culture, and recognized by a UCL Provost's Student Award for Public Engagement. Project dissemination was supported by the National Institute for Health Research (NIHR) Biomedical Research Centre at Moorfields Eye Hospital NHS Foundation Trust and UCL Institute of Ophthalmology. Particular thanks go to Laura Cream, Richard Cable and Andi Skilton for professional support and advice with public engagement and project delivery. The views expressed are those of the authors and not necessarily those of UCL, the NHS, the NIHR or the Department of Health.

We would like to thank the members of the Eating for Eye Health focus group who wholeheartedly engaged with the focus group discussion and/or the community kitchen cookery day; Susana S. Couto Irving for expert nutritional advice and recipe adaptation for macular health; Siobhan Mannion from the Manor Gardens Community Kitchen Project, who managed the cookery day; Chrishne Sivapathasuntharam and Garima Sharma, PhD research students at UCL Institute of Ophthalmology, who helped extensively 'behind the scenes', facilitating at the focus group and the community cookery day; Zubin Saihan, Consultant Ophthalmologist, Moorfields Eye Hospital, and Melanie Dani, SpR in Care of the Elderly Medicine, who presented 'expert' opinions at the focus group; professional videographer and photographers Emily Packer (Juniper Mountain Films) and Silvio Palladino, who created high-quality, colourful multimedia from the cookery day; Pod City Road healthy food bar for hosting the Eating for Eye Health focus group; and the Central Street Cookery School at St Luke's 
Community Centre, Clerkenwell for providing the kitchen space for the cookery day at a community rate. Finally, many thanks to the NCCPE for recognition with the Engage Award (November 2016) and ongoing support, which will facilitate development of the engagement approach and the project aims.

\section{Notes on the contributors}

Rose Gilbert is an Honorary Clinical Fellow at Moorfields Eye Hospital, London. She has postgraduate research degrees in ophthalmology and immunology, and health psychology from UCL, London. After completing the Train and Engage publicengagement course during her PhD studies at $\mathrm{UCL}$, she was inspired to initiate Eating for Eye Health to address the unmet needs of patients in NHS eye clinics.

Ann Rawlings is a retired teacher and Chartered Fellow of the Institute of Horticulture, who judges for London in Bloom and the National Garden Society, and mentors Royal Parks apprentices. She was diagnosed with dry AMD 15 years ago, and has experienced a gradual deterioration in her eyesight since then. Ann continues to grow a wide variety of fruit and vegetables in her garden allotment, an interest that immediately attracted her to become involved in the Eating for Eye Health project.

Michael Dixon is a senior GP partner at the Culm Valley Integrated Centre for Health, Devon; Chair of the College of Medicine and Integrated Health; and Medical Advisor to HRH The Prince of Wales. He has an interest in integrated health and public policy, is the National Clinical Lead for Social Prescription at NHS England and holds visiting professorships at UCL and the University of Westminster.

Ana Rita Gonçalves de Pinho is completing a PhD in cornea tissue engineering at UCL. With a background in biomedical engineering, she usually defines herself as an engineer by training and a biologist by heart, passionate about education, public engagement, science policy and event planning. She also undertakes outreach work with minority groups, encouraging interest in STEM subjects.

Tadhg Caffrey was Public Engagement Manager (SLMS) within UCL Culture. He is focused on establishing mutually beneficial modes of engagement, and in supporting UCL staff and students in their engagement work. He is particularly interested in questioning how engagement can create tangible change for community and public groups, and how this in turn can change academic agendas and practice.

\section{References}

Age-Related Eye Disease Study Research Group (2001) 'A randomized, placebo-controlled, clinical trial of high-dose supplementation with vitamins $C$ and $E$, beta carotene, and zinc for age-related macular degeneration and vision loss (AREDS Report No. 8)'. Archives of Ophthalmology, $119,1417-36$.

Anchor \& Magnet (2012) 'Conversation Dinner / 2012'. Online. www.anchorandmagnet.org/blankes7np (accessed 2 June 2019).

Brandling, J. and House, W. (2009) 'Social prescribing in general practice: Adding meaning to medicine'. British Journal of General Practice, 59 (563), 454-6.

Clare, A. (2001) Psychiatry in Dissent: Controversial issues in thought and practice. 2nd ed. London: Routledge.

Cohen-Mansfield, J. and Perach, R. (2015) 'Interventions for alleviating loneliness among older persons: A critical review'. American Journal of Health Promotion, 29 (3), e109-25.

Coulson, A. (2014) 'Food in community engagement: If food be the tool of the Gods, then engage on!'. commsgodigital, 10 September. Online. https://tinyurl.com/yy67gjss (accessed 22 May 2019). 
Dagnelie, G. (2013) 'Age-related psychophysical changes and low vision'. Investigative Ophthalmology and Visual Science, 54 (14), ORSF88-93.

Dawn, A.G., Santiago-Turla, C. and Lee, P.P. (2003) 'Patient expectations regarding eye care: Focus group results'. Archives of Ophthalmology, 121 (6), 762-8.

Dean, S., Mathers, J.M., Calvert, M., Kyte, D.G., Conroy, D., Folkard, A., Southworth, S., Murray, P.I. and Denniston, A.K. (2017) "'The patient is speaking": Discovering the patient voice in ophthalmology'. British Journal of Ophthalmology, 101 (6), 700-8.

Evans, J.R. and Lawrenson, J.G. (2017a) 'Antioxidant vitamin and mineral supplements for slowing the progression of age-related macular degeneration'. Cochrane Database of Systematic Reviews, 7, Article CD000254, 1-128. Online. https://tinyurl.com/yykrxkkh (accessed 22 May 2019).

Evans, J.R. and Lawrenson, J.G. (2017b) 'Antioxidant vitamin and mineral supplements for preventing age-related macular degeneration'. Cochrane Database of Systematic Reviews, 7 , Article CD000253, 1-64. Online. https://tinyurl.com/y6e3dglw (accessed 22 May 2019).

Garcia, A.L., Reardon, R., Hammond, E., Parrett, A. and Gebbie-Diben, A. (2017) 'Evaluation of the "Eat Better Feel Better" cooking programme to tackle barriers to healthy eating'. International Journal of Environmental Research and Public Health, 14 (4), Article 380, 1-18. Online. www.mdpi.com/1660-4601/14/4/380 (accessed 22 May 2019).

Gilbert, R.M., Rawlings, A., Dixon, M., Couto Irving, S.S., Sivaprasad, S. (2018) 'Dietary change for macular health of older people: Eating for Eye Health, a pilot community kitchens intervention', The Lancet, 392 (S37).

Herbert, J., Flego, A., Gibbs, L., Waters, E., Swinburn, B., Reynolds, J. and Moodie, M. (2014) 'Wider impacts of a 10-week community cooking skills program - Jamie's Ministry of Food, Australia'. BMC Public Health, 14, Article 1161, 1-14. Online. https://tinyurl.com/y6chuavv (accessed 22 May 2019).

Hogg, R.E., Woodside, J.V., McGrath, A., Young, I.S., Vioque, J.L., Chakravarthy, U., De Jong, P.T., Rahu, M., Seland, J., Soubrane, G., Tomazzoli, L., Topouzis, F. and Fletcher, A.E. (2017) 'Mediterranean Diet Score and its association with age-related macular degeneration: The European Eye Study'. Ophthalmology, 124 (1), 82-9.

Huber, M., Van Vliet, M., Giezenberg, M., Winkens, B., Heerkens, Y., Dagnelie, P.C. and Knottnerus, J.A. (2016) 'Towards a "patient-centred" operationalisation of the new dynamic concept of health: A mixed methods study'. BMJ Open, 6 (1), Article e010091, 1-11. Online. https://bmjopen.bmj.com/content/6/1/e010091 (accessed 22 May 2019).

lacovou, M., Pattieson, D.C., Truby, H. and Palermo, C. (2013) 'Social health and nutrition impacts of community kitchens: A systematic review'. Public Health Nutrition, 16 (3), 535-43.

Kimberlee, R.H. (2013) Developing a Social Prescribing Approach for Bristol: Project report. Bristol: Bristol Health and Wellbeing Board.

Kimberlee, R. (2016) 'What is the value of social prescribing?'. Advances in Social Sciences Research Journal, 3 (3), 29-35.

Matthews, K., Nazroo, J. and Whillans, J. (2017) 'The consequences of self-reported vision change in later-life: Evidence from the English Longitudinal Study of Ageing'. Public Health, 142, 7-14.

Mendes de Leon, C.F. (2005) 'Social engagement and successful aging'. European Journal of Ageing, 2 (1), 64-6.

Michie, S., Atkins, L. and West, R. (2014) The Behaviour Change Wheel: A guide to designing interventions. London: Silverback.

Michie, S., van Stralen, M.M. and West, R. (2011) 'The behaviour change wheel: A new method for characterising and designing behaviour change interventions'. Implementation Science, 6, Article 42, 1-11. Online. https://implementationscience.biomedcentral.com/track/ pdf/10.1186/1748-5908-6-42 (accessed 26 June 2019).

NICE (National Institute for Health and Care Excellence) (2016) Community Engagement: Improving health and wellbeing and reducing health inequalities (NICE Guideline NG44). London: National Institute for Health and Care Excellence.

Nollett, C.L., Bray, N., Bunce, C., Casten, R.J., Edwards, R.T., Hegel, M.T., Janikoun, S., Jumbe, S.E., Ryan, B., Shearn, J., Smith, D.J., Stanford, M., Xing, W. and Margrain, T.H. (2016) 'High prevalence of untreated depression in patients accessing low-vision services'. Ophthalmology, 123 (2), 440-1.

Ozbay, F., Johnson, D.C., Dimoulas, E., Morgan, C.A., Charney, D. and Southwick, S. (2007) 'Social support and resilience to stress: From neurobiology to clinical practice'. Psychiatry (Edgmont), $4(5), 35-40$. 
Polley, M., Bertotti, M., Kimberlee, R., Pilkington, K. and Refsum, C. (2017) A Review of the Evidence Assessing Impact of Social Prescribing on Healthcare Demand and Cost Implications: Technical report. London: University of Westminster.

Steinman, B.A. and Vasunilashorn, S. (2011) 'Biological risk of older adults with visual impairments'. Journal of Nutrition, Health and Aging, 15 (4), 296-302.

Stevens, R., Bartlett, H. and Cooke, R. (2015) 'Dietary analysis and nutritional behaviour in people with and without age-related macular disease'. Clinical Nutrition ESPEN, 10 (3), e112-17.

Tolman, J., Hill, R.D., Kleinschmidt, J.J. and Gregg, C.H. (2005) 'Psychosocial adaptation to visual impairment and its relationship to depressive affect in older adults with age-related macular degeneration'. Gerontologist, 45 (6), 747-53.

Twamley, K., Evans, J. and Wormald, R. (2011) 'Why involve consumers in eye health research?'. Eye, 25 (8), 969-70.

VisionUK (2016) 'Macular Society's call for more funding of research into macular degeneration secures leading expert endorsement'. Online. www.visionuk.org.uk/58012-2/ (accessed 26 June 2019).

WHO (World Health Organization) (2019) 'Constitution of WHO: Principles'. Online. http://origin. who.int/about/mission/en/ (accessed 26 June 2019).

Wu, J., Cho, E., Willett, W.C., Sastry, S.M. and Schaumberg, D.A. (2015) 'Intakes of lutein, zeaxanthin, and other carotenoids and age-related macular degeneration during 2 decades of prospective follow-up'. JAMA Ophthalmology, 133 (12), 1415-24. 